\title{
Feasibility study of artifacts on the neutron logging curve near the boundary of layers
}

\author{
Urszula Wiącek $^{1}$ [D $\cdot$ Urszula Woźnicka $^{1}$
}

Received: 8 May 2019 / Accepted: 27 June 2019 / Published online: 6 July 2019

(c) The Author(s) 2019

\begin{abstract}
The neutron borehole probe equipped with a neutron source and a system of neutron detectors is commonly used to determine porosity of geological formations. Transport of neutrons through media relies on their interaction with atomic nuclei, mainly with the process of scattering and absorption. Curves recorded by detectors for a given geological layer bordered by a medium with other neutron properties form the so-called anomalies. The course of the anomaly mainly depends on neutron properties of the bordering media and also on the measurement geometry, borehole properties, etc. The paper focuses mainly on an influence of neutron properties of bordered rock media on the course of the anomaly curve, especially near the media boundaries. In some cases of bordering media, characterized by specific neutron properties, the course of anomalies can be significantly disturbed, forming specific artifacts. The paper explains that the presence of artifacts is closely related to the properties of bordered media for both fast and thermal neutrons, i.e., the slowing down length of fast neutrons and the absorption cross section of thermal neutrons. Artifacts can be the cause of misinterpretation of the neutron rock porosity probe measurement. All considerations presented in this paper are based on results obtained from MC calculations performed using MCNP5 code (MCNP Team in X-5 Monte Carlo Team, MCNP—a general Monte Carlo N-particle transport code version 5. Los Alamos National Laboratory LA-UR-03-1987, 2008).
\end{abstract}

Keywords Neutron logging $\cdot$ MCNP modeling $\cdot$ Boundary of layers $\cdot$ Neutron transport parameters $\cdot$ Neutron artifacts

\section{Introduction}

Neutron measurement techniques are widely used in profiling of geophysical exploration wells. The neutron borehole probe used to determine the rock medium porosity is equipped with a neutron source and a system of detectors. Neutrons reach the detectors after passing through the studied rock medium. Detectors counts, after applying an appropriate calibration and interpretation procedures, give information on the rock medium porosity. From the point of view of the neutron transport physics in media, we deal with an interaction of neutrons with atomic nuclei, mainly with the scattering and absorption processes. It causes the reduction in neutron energy and their final absorption. These phenomena cause that a small number of neutrons from the source reach the detectors, and their number depends on

Urszula Wiącek

urszula.wiacek@ifj.edu.pl

1 Institute of Nuclear Physics Polish Academy of Sciences, Radzikowskiego 152, 31-342 Kraków, Poland neutron properties of the medium. A typical shape of the curve (the so-called anomaly), recorded by the detector for two layers of different neutron properties bordering each other, changes monotonically (see later, e.g., Fig. 2). The shape of the anomaly depends mainly on neutron properties of the bordering media. In order to determine the relationship between the course of anomalies and neutron properties of adjacent media, we use integral neutron parameters, mainly the slowing down length of fast neutrons $\left(L_{\mathrm{s}}\right.$, marked in figures Ls) and the macroscopic absorption cross section of thermal neutrons ( $\Sigma_{a}$, marked in figures Sigma).

However, it should be noted that the transport of neutrons from the source to the detector also leads through the construction materials of the probe, the borehole filled with drilling mud and through the intermediate zone between the borehole wall and the rock formation under study. The probe design itself, mainly a type of the source and the sourcedetector distance, also plays an important role. The paper focuses only on an influence of neutron parameters of bordered rock media on the course of the anomaly curve, especially near the media boundaries. 
In some cases of bordering media characterized by specific neutron properties, the course of anomalies can be significantly disturbed. Similar disturbances in the anomalies at the boundary of layers were observed in the paper (Gardner et al. 2007), and they have been described as the effect of numerical errors. The analyses carried out in this work prove that the appearance of the mentioned artifacts is a result of specific energy distributions of neutron fields in the boundary regions of layers with much different neutron parameters. These disorders, called artifacts, may be the cause of misinterpretation of neutron measurements.

\section{Numerical simulations of anomalies recorded by the neutron probe}

A numerical method was used to investigate the effect of artifacts appearance on the curves registered by the neutron probe. For the Monte Carlo modeling and simulations of the neutron transport, MCNP code was used (MCNP 2008; Wiącek and Woźnicka 2016). The responses of the neutron probe detectors were calculated under strictly defined measurement conditions. It was assumed that the probe was equipped with an isotope source of fast neutrons, Am-Be, and sets of thermal and epithermal detectors at the distances of $L_{1}=31 \mathrm{~cm}$ and $L_{2}=54.5 \mathrm{~cm}$ from the source. The probe was placed eccentrically in the borehole and was adhered to the wall. The borehole of diameter $D=21.6 \mathrm{~cm}$ was filled with water. Detector responses in model geological formations of specific chemical composition and known neutron parameters were investigated. The properties and parameters of the rock models are summarized in Table 1. Elemental
Table 2 Oxide and elemental composition (wt $\%$ ) of rock matrix of models

\begin{tabular}{|c|c|c|c|c|c|}
\hline & M & BW & KW & L13 & MUD \\
\hline $\mathrm{H}_{2} \mathrm{O}$ & 1.200 & & & & 1.243 \\
\hline $\mathrm{CO}_{2}$ & 8.000 & 0.657 & 0.605 & 0.707 & 3.625 \\
\hline $\mathrm{Na}_{2} \mathrm{O}$ & - & 0.292 & 1.450 & 0.327 & 0.518 \\
\hline $\mathrm{MgO}$ & - & 0.409 & 0.294 & 0.491 & 3.107 \\
\hline $\mathrm{Al}_{2} \mathrm{O}_{3}$ & 7.000 & 36.790 & 25.184 & 33.664 & 13.465 \\
\hline $\mathrm{SiO}_{2}$ & 72.500 & 58.472 & 68.562 & 61.475 & 59.039 \\
\hline $\mathrm{K}_{2} \mathrm{O}$ & 1.800 & 0.974 & 2.292 & 1.320 & 3.625 \\
\hline $\mathrm{CaO}$ & 7.500 & 0.838 & 0.771 & 0.900 & 4.661 \\
\hline $\mathrm{Fe}_{2} \mathrm{O}_{3}$ & 2.000 & 1.568 & 0.842 & 1.115 & - \\
\hline $\mathrm{TiO}_{2}$ & - & - & - & - & 0.621 \\
\hline $\mathrm{MnO}$ & - & - & - & - & 0.124 \\
\hline $\mathrm{Fe}_{2} \mathrm{O}_{3}$ & - & - & - & - & 5.697 \\
\hline $\mathrm{Li}$ & - & - & - & - & 0.004 \\
\hline B & a) & - & - & - & 0.013 \\
\hline $\mathrm{C}$ & - & - & - & - & 2.486 \\
\hline$S$ & - & - & - & - & 1.657 \\
\hline $\mathrm{Cl}$ & - & - & - & - & 0.104 \\
\hline $\mathrm{Ni}$ & - & - & - & - & 0.009 \\
\hline $\mathrm{Sm}$ & - & - & - & - & 0.001 \\
\hline $\mathrm{Gd}$ & - & - & - & - & 0.001 \\
\hline
\end{tabular}

M-rock matrix of models M1-M6, MUD—rock matrix of models MUD2, MUD5, MUD9, MUD15 and BW, KW, L13 - ceramic standards of the calibration station of Geofizyka Kraków in Zielona Góra (Wiącek and Woźnicka 2016)

a) ${ }^{10} \mathrm{~B}=0.00163 \mathrm{wt} \%$ to obtain $\Sigma_{\text {a matrix }}=11.1$ c.u. ${ }^{10} \mathrm{~B}=0.00663 \mathrm{wt} \%$ to obtain $\Sigma_{\text {a matrix }}=24.2$ c.u.

compositions of the matrix of the rock models are presented in Table 2. The pores of rock models were filled with water.
Table 1 Porosity and neutron parameters of rock models

\begin{tabular}{lclcc}
\hline Rock model & Porosity $\phi(\%)$ & $\begin{array}{l}\text { Macroscopic absorption } \\
\text { cross section } \Sigma_{\mathrm{a}}(\mathrm{c} . \mathrm{u} .)\end{array}$ & $\begin{array}{l}\text { Slowing down } \\
\text { length } L_{\mathrm{s}}(\mathrm{cm})\end{array}$ & $\begin{array}{l}\text { Density } \rho \\
\left(\mathrm{g} / \mathrm{cm}^{3}\right)\end{array}$ \\
\hline M1 & 7.5 & 12.63 & 14.57 & 2.51 \\
M2 & 7.5 & 28.27 & 14.57 & 2.51 \\
M3 & 20.0 & 13.89 & 11.48 & 2.30 \\
M4 & 20.0 & 29.18 & 11.48 & 2.30 \\
M5 & 45.0 & 15.72 & 8.97 & 1.90 \\
M6 & 45.0 & 27.04 & 8.97 & 1.90 \\
Mud2 & 2.0 & 22.65 & 17.14 & 2.65 \\
Mud5 & 5.0 & 23.86 & 15.32 & 2.60 \\
Mud9 & 9.0 & 24.70 & 13.73 & 2.53 \\
Mud15 & 15.0 & 25.20 & 12.20 & 2.43 \\
BW & 23.8 & 11.28 & 9.63 & 2.12 \\
KW & 16.6 & 9.86 & 10.91 & 2.20 \\
L13 & 60.0 & 17.13 & 7.42 & 1.64 \\
Water & 100.0 & 19.30 & 7.01 & 1.00 \\
\hline
\end{tabular}

M-rock matrix of models M1-M6, MUD—rock matrix of models MUD2, MUD5, MUD9, MUD15 and BW, KW, L13 - ceramic standards of the calibration station of Geofizyka Kraków in Zielona Góra (Wiącek and Woźnicka 2016) 
It was assumed that a given horizontal layer of the model formation with a thickness $H$ was surrounded on both sides by a layer (at least $50 \mathrm{~cm}$ thick) with different neutron

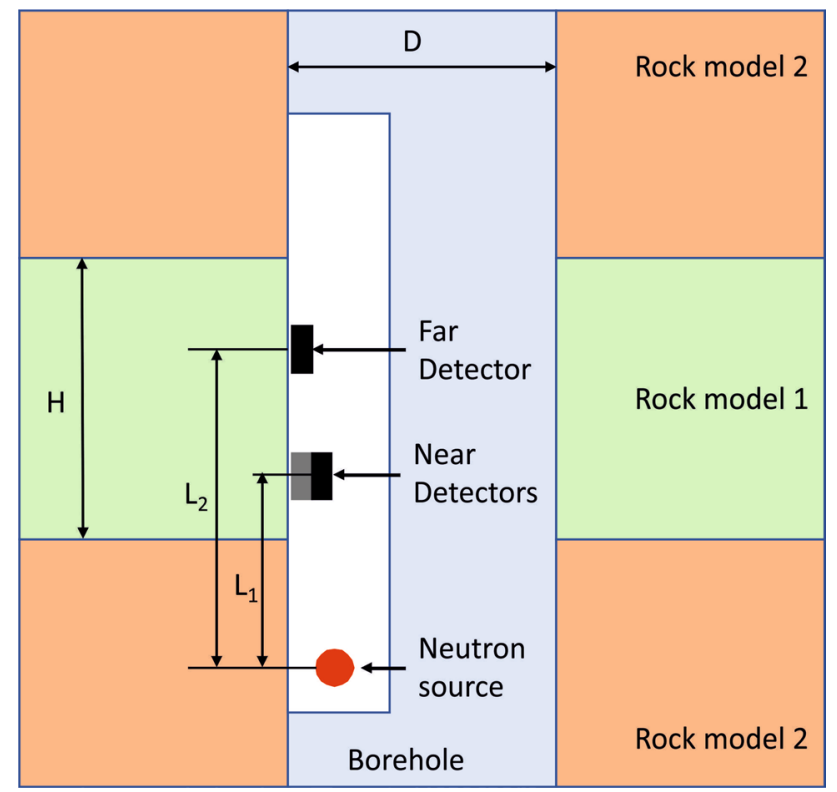

Fig. 1 The scheme of the measurement geometry. Near detectors are dedicated to measure thermal and epithermal neutrons. Far detector is dedicated to measure epithermal neutrons parameters. The layers were cut with borehole perpendicularly. A scheme of the measurement geometry is shown in Fig. 1. A detailed description of the numerical calculations can be found in the following articles (Wiącek 2018; Wiącek and Dworak 2018).

\section{Typical courses of anomalies in rock models}

A complete list of the numerical calculations for layers of all combinations of rock models is collected in the papers (Woźnicka et al. 2012; Wiącek 2018). Here only a few examples of the anomalies are presented. Figure 2 presents the responses of three detectors of the probe: near thermal neutron detector (Fig. 2a) and epithermal near and far detectors (Fig. $2 b$ and c). The anomaly corresponds to the rock model M6 of thickness $50 \mathrm{~cm}$ surrounded on both sides with the rock model M1. All anomalies registered by three detectors maintain the monotonic course, corresponding to the neutron properties of the M1 and M6 adjacent media.

The horizontal axis shows the position of the near (or far, in Fig. 2c) detector in relation to the left boundary of the layer. It means that if the near detector is in the position $x=0 \mathrm{~cm}$, the source of neutrons is in the position $x=L_{1}=-31 \mathrm{~cm}$. In areas where both the source and the detector are located in the same rock medium, the detector's signal reaches the characteristic
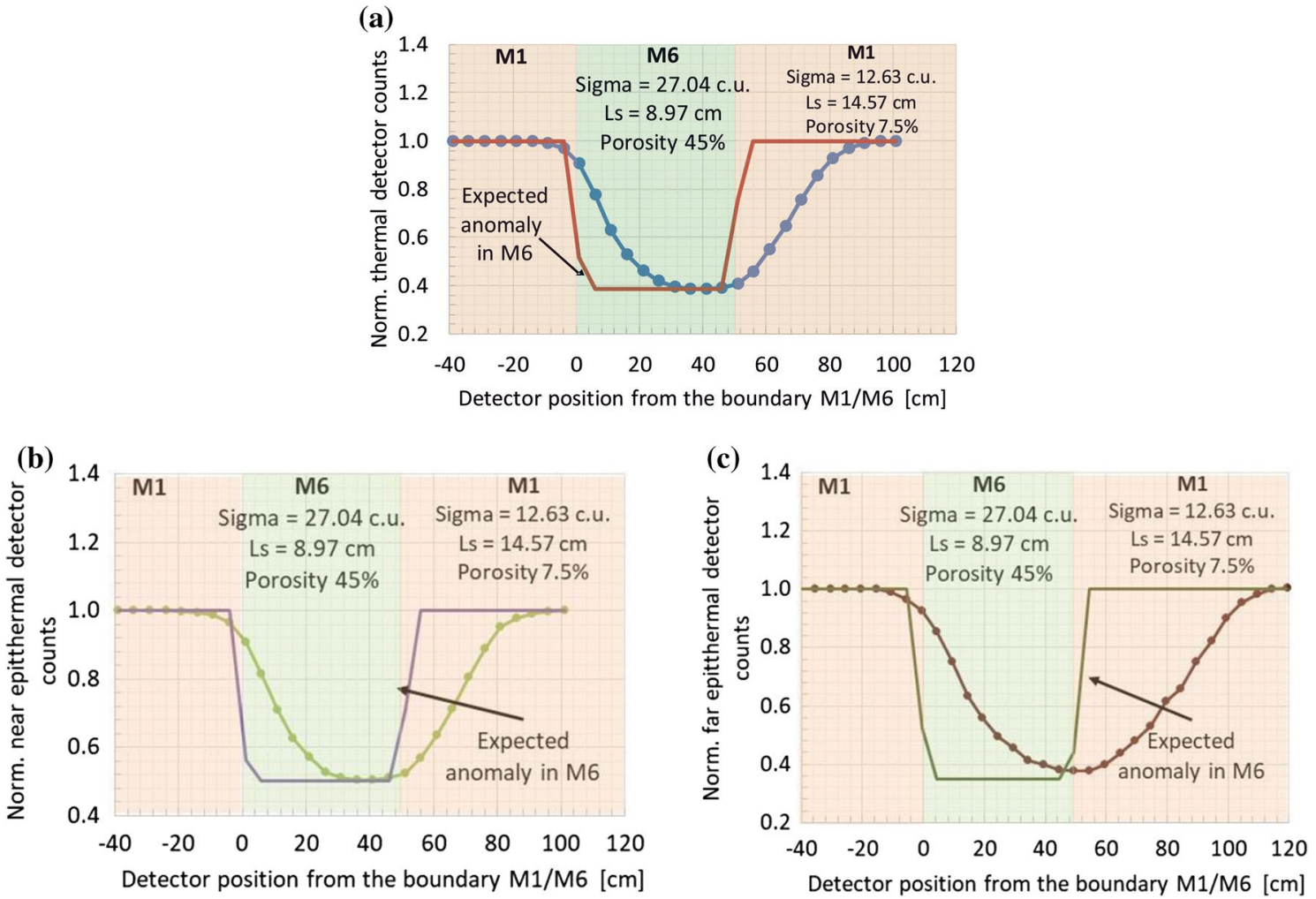

Fig. 2 Typical anomaly registered by three detectors of the neutron probe. Example for rock model layer M6 of thickness $H=50 \mathrm{~cm}$ surrounded with rock model M1: a near thermal neutron detector, $\mathbf{b}$ near epithermal neutron detector and $\mathbf{c}$ far epithermal neutron detector 

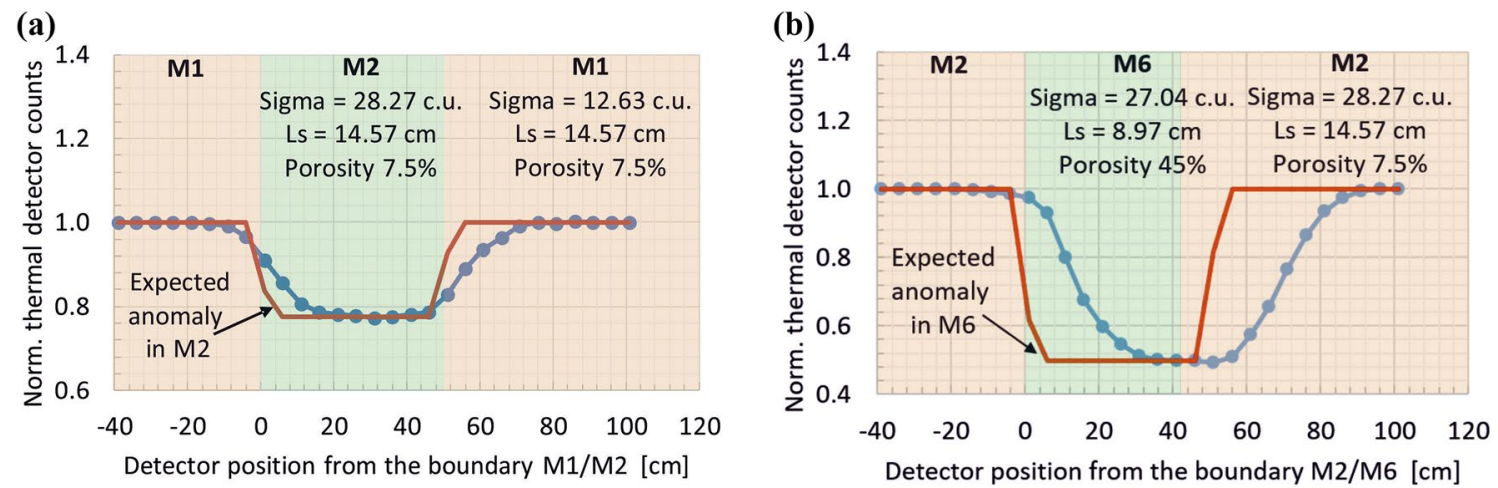

Fig. 3 Courses of anomalies registered by the near thermal neutron detector for bordering rock layers which differ significantly only with one neutron parameter: a Case I: in thermal neutron absorption cross section $\Sigma_{\mathrm{a}}$, b Case II: in fast neutron slowing down length, $L_{\mathrm{s}}$

value for a given medium. The charts show the count values normalized to the detector response in the rock medium which surrounds the layer.

Figure 3 presents two examples of anomalies for adjacent media which differ in one of the neutron parameters. Case I presents the anomaly for rock model M2 of thickness $H=50 \mathrm{~cm}$ surrounded on both sides by rock model M1. The models M1 and M2 differ significantly in values of the absorption cross sections, $\Sigma_{\mathrm{a}}$, while maintaining the same values of the fast neutron slowing down length, $L_{\mathrm{s}}$. Case II presents the neutron anomaly for a layer of model M6 surrounded by rock model M2. The models M2 and M6 differ significantly in $L_{\mathrm{s}}$ and have similar values of $\Sigma_{\mathrm{a}}$.

\section{Examples of the artifacts}

It has been shown above that typical, monotonic anomalies are recorded when neighboring layers have different neutron parameters, $L_{\mathrm{s}}$ and $\Sigma_{\mathrm{a}}$. However, for several combinations of the adjacent layers from the base of models collected in Table 1, abnormal courses of the anomalies with artifacts are observed. Anomalies with the artifacts are shown in Figs. 4, 5,6 and 7. It was observed that artifacts appear only on the thermal neutron detector responses for the media which differ significantly in $\Sigma_{\mathrm{a}}$ and $L_{\mathrm{s}}$.

To explain the phenomenon, let us focus on the example presented in Fig. 4. The horizontal axis corresponds to the position of the detector in relation to the layer boundary. When the detector is in the position $x=0 \mathrm{~cm}$, the Am-Be source is still in the previous layer at the point $x=-31 \mathrm{~cm}$.

Knowing $L_{\mathrm{s}}$, the average distance $\langle r\rangle$ (in a straight line) between the source position and the point when the neutron is slowed down to a thermal energy can be estimated as:

$\langle r\rangle=\sqrt{6} L_{s}$

The medium M2 has $L_{\mathrm{s}}=14.57 \mathrm{~cm}$ and $\langle r\rangle=35.7 \mathrm{~cm}$. It means that the fast neutrons generated by the source to the

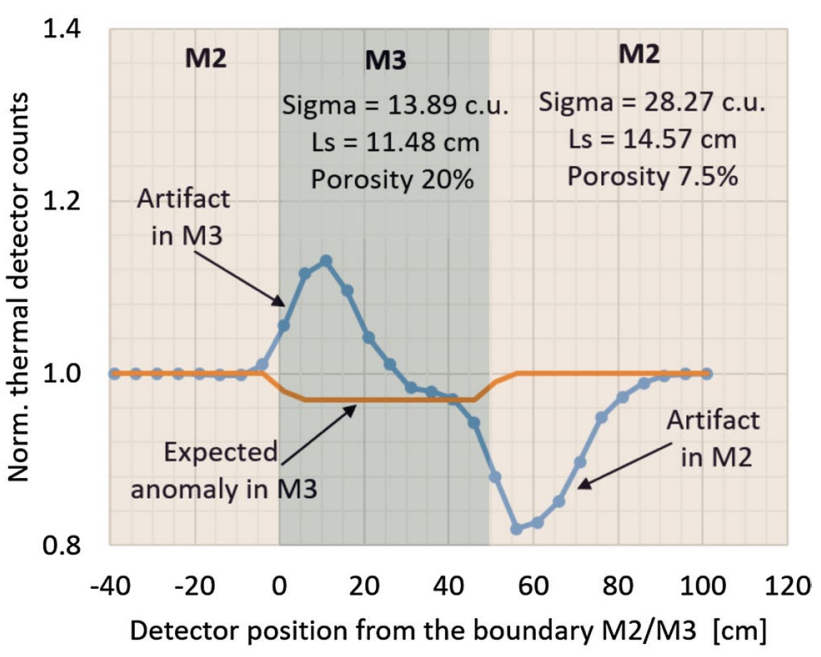

Fig. 4 Abnormal courses of anomalies with artifacts registered by the near thermal neutron detector for bordering rock layers M2/M3. $L_{\mathrm{s}}(\mathrm{M} 2)>L_{\mathrm{s}}(\mathrm{M} 3)$ and $\Sigma_{\mathrm{a}}(\mathrm{M} 2)>\Sigma_{\mathrm{a}}(\mathrm{M} 3)$

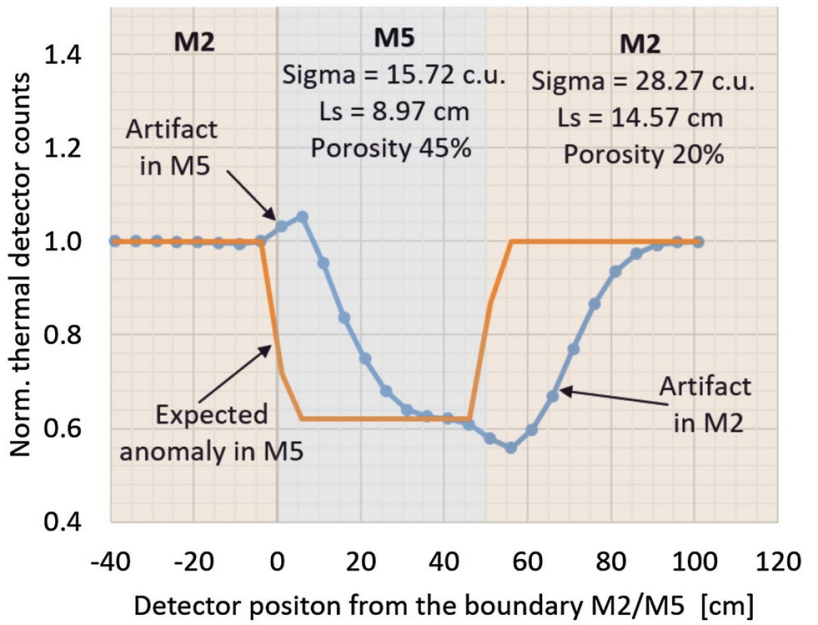

Fig. 5 Abnormal courses of anomalies with artifacts registered by the near thermal neutron detector for bordering rock layers M2/M5. $L_{\mathrm{s}}(\mathrm{M} 2)>L_{\mathrm{s}}(\mathrm{M} 5)$ and $\Sigma_{\mathrm{a}}(\mathrm{M} 2)>\Sigma_{\mathrm{a}}(\mathrm{M} 5)$ 


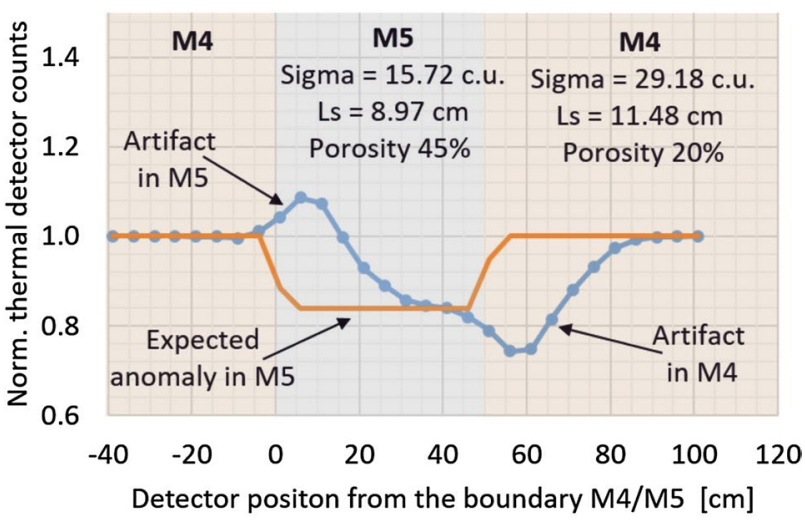

Fig. 6 Abnormal courses of anomalies with artifacts registered by the near thermal neutron detector for bordering rock layers M4/M5. $L_{\mathrm{s}}(\mathrm{M} 4)>L_{\mathrm{s}}(\mathrm{M} 5)$ and $\Sigma_{\mathrm{a}}(\mathrm{M} 4)>\Sigma_{\mathrm{a}}(\mathrm{M} 5)$

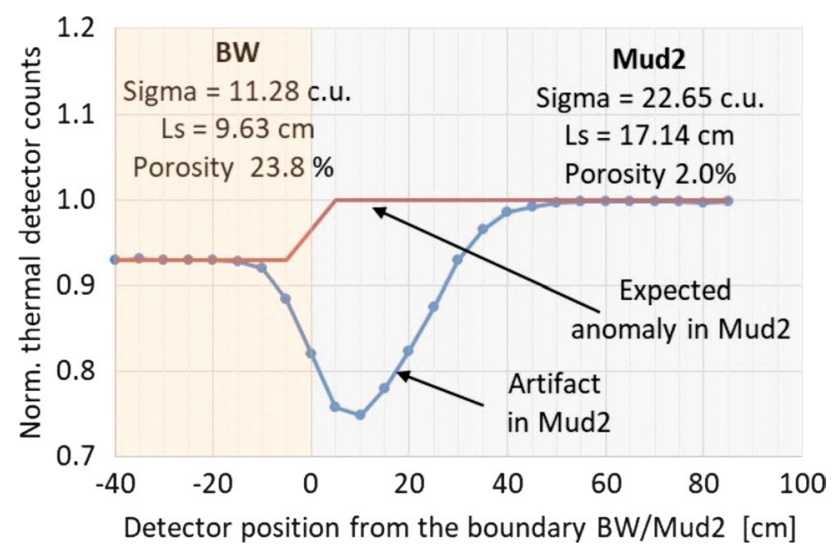

Fig. 7 Abnormal courses of anomalies with artifacts registered by the near thermal neutron detector for bordering rock layers BW/Mud2. $L_{\mathrm{s}}(\mathrm{Mud} 2)>L_{\mathrm{s}}(\mathrm{BW})$ and $\Sigma_{\mathrm{a}}(\operatorname{Mud} 2)>\Sigma_{\mathrm{a}}(\mathrm{BW})$

area of M2 may appear in the area of layer M3. Up to about $5 \mathrm{~cm}$ from the boundary with M2, the fast neutrons are thermalized and can reach the detector. The M3 medium weakly absorbs neutrons; therefore a significant number of neutrons accumulate close to the border, forming the artifact. For the position $50 \mathrm{~cm}<x<80 \mathrm{~cm}$, the neutron source is still in M3 while the detector is already in M2. There is a very strong neutron absorption in M2 near the boundary with M3. In this area $(50 \mathrm{~cm}<x<80 \mathrm{~cm})$, there are neutrons which started their travel in M3, were partially slowed down and as such were easily absorbed in M2, unable to reach the detector anymore. This causes a significant loss of registered particles in the area of $50 \mathrm{~cm}<x<80 \mathrm{~cm}$.

Two types of artifacts are observed:

Type I. The case when the probe moves from the medium for which we expect a higher number of counts to the medium in which the number of counts is lower. The artifact appears in the area of the medium with a lower number of counts, close to the boundary for $0<x<20 \mathrm{~cm}$, and is characterized by a significant overstating of the counts number than expected.

Type II. Reverse case-movement of the probe is from the medium of a lower count rate to the medium of a higher count rate. The artifact appears in the area of the medium with a higher count number, close to the boundary for $50 \mathrm{~cm}<x<80 \mathrm{~cm}$, and is characterized by a significant understating of counts than expected.

\section{Analysis of occurrence of the artifacts}

\section{Differences in the neutron parameters of bordering media and the presence of artifacts}

Appearance of an artifact is affected by the neutron parameters of neighboring media. Let us consider the media boundary around the detector location $x=0 \mathrm{~cm}$. The probe moves from left to right. In all four cases observed, it was found that the first medium is characterized by a higher $L_{\mathrm{s}}$ value and a higher $\Sigma_{\mathrm{a}}$ value than those of the next medium. This causes an appearance of the type I artifact. In the opposite situation (around the detector location $x=50 \mathrm{~cm}$ ), when the first medium has lower values of parameters $L_{\mathrm{s}}$ and $\Sigma_{\mathrm{a}}$ than the next one, a type II artifact appears.

Fig. 4: $\quad L_{\mathrm{s}}(\mathrm{M} 2) / L_{\mathrm{s}}(\mathrm{M} 3)=1.27 \quad \Sigma_{\mathrm{a}}(\mathrm{M} 2) / \Sigma_{\mathrm{a}}(\mathrm{M} 3)=2.4$

Fig. 5: $\quad L_{\mathrm{s}}(\mathrm{M} 2) / L_{\mathrm{s}}(\mathrm{M} 5)=1.62 \quad \Sigma_{\mathrm{a}}(\mathrm{M} 2) / \Sigma_{\mathrm{a}}(\mathrm{M} 5)=1.80$

Fig. 6: $\quad L_{\mathrm{s}}(\mathrm{M} 4) / L_{\mathrm{s}}(\mathrm{M} 5)=1.28 \quad \Sigma_{\mathrm{a}}(\mathrm{M} 4) / \Sigma_{\mathrm{a}}(\mathrm{M} 5)=1.86$

Fig. 7: $\quad L_{\mathrm{s}}(\operatorname{Mud} 2) / L_{\mathrm{s}}(\mathrm{BW})=1.78 \quad \Sigma_{\mathrm{a}}(\operatorname{Mud} 2) / \Sigma_{\mathrm{a}}(\mathrm{BW})=2.01$

The analysis of all considered cases of the simulated anomalies for all combinations of adjacent layers (Table 1) showed that only in the case of the above four combinations of layers M2/M3, M2/M5, M4/M5 and Mud2/BW, there are such relationships between the $L_{\mathrm{s}}$ and $\Sigma_{\mathrm{a}}$ parameters. It can be clearly stated that appearance:

Artifacts type $1, \quad$ when $L_{\mathrm{s} 1} / L_{\mathrm{s} 2}>1$ and $\Sigma_{\mathrm{a} 1} / \Sigma_{\mathrm{a} 2}>1$

Artifacts type II, when $L_{\mathrm{s} 1} / L_{\mathrm{s} 2}<1$ and $\Sigma_{\mathrm{a} 1} / \Sigma_{\mathrm{a} 2}<1$

It was shown above that the bounded media which differ only with one parameter $\left(L_{\mathrm{s}}\right.$ or $\Sigma_{\mathrm{a}}$, see Fig. 2$)$ do not create an artifact. To be sure, it should be assumed that the artifact appears when the parameters of one medium $\left(L_{\mathrm{s}}\right.$ and $\left.\Sigma_{\mathrm{a}}\right)$ differ by at least $20 \%$. 
Let us consider two cases for which artifacts are not observed:

1. $\quad L_{\mathrm{s}}(\mathrm{M} 4) / L_{\mathrm{s}}(\mathrm{M} 6)=1.28$ but $\Sigma_{\mathrm{a}}(\mathrm{M} 4) / \Sigma_{\mathrm{a}}(\mathrm{M} 6)=1.08$

2. $\Sigma_{\mathrm{a}}(\operatorname{Mud} 15) / \Sigma_{\mathrm{a}}(\mathrm{KW})=2.56 \quad$ but $\quad L_{\mathrm{s}}(\operatorname{Mud} 15) / L_{\mathrm{s}}(\mathrm{KW})=1.12$

For both cases, one of the parameter: $\Sigma_{\mathrm{a}}$ or $L_{\mathrm{s}}$, of one of the medium differs less than $20 \%$. For the first case, M4/ M6, the ratios of $\Sigma_{\text {a }}$ differ only by $8 \%$, and for the second case, Mud15/KW, the ratios of the slowing down length differ only by $12 \%$.

\section{Energetic and spatial distribution of the neutron field near boundary of layers}

The phenomenon of the appearance of artifacts should be explained by analyzing the energetic and spatial distribution of the neutron field between the neutron source and the detection point.

Detailed analysis of the observed phenomenon can be performed by analyzing the detection conditions of the thermal neutron detector. The detector's signal is generated as a result of neutron absorption in its volume. In the examples discussed, the thermal neutron detector was a helium counter shielded by cadmium layer with the window toward the rock formation. A detailed description of this neutron detection system can be found in Wiącek (2018). The total detector signal is formed by neutrons from a particular range of their energy spectrum. Two parts of this spectrum can be distinguished with numerical calculations: E1 for a low-energy component with the maximum at energy $E<0.4 \mathrm{eV}$ and $\mathrm{E} 2$ for a higher-energy component with the maximum at energy $E>0.4 \mathrm{eV}$.

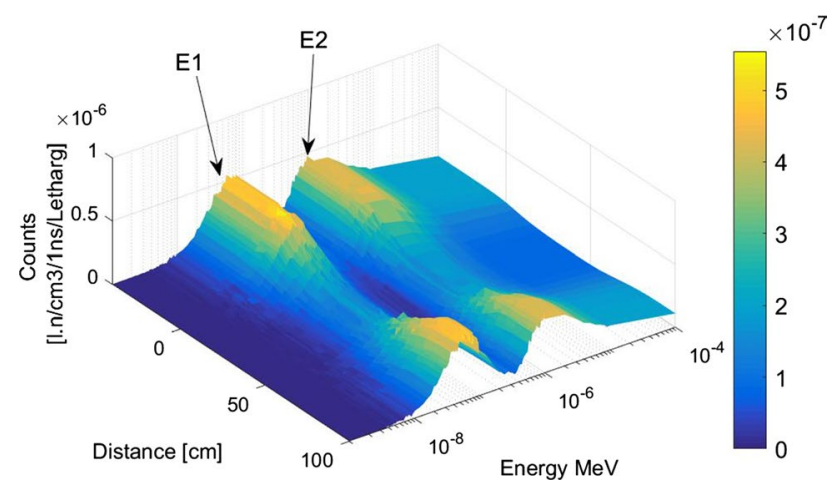

Fig. 8 Neutron energy-spatial distribution forming the response of the near thermal detector that registers the anomaly for the rock model M6 of thickness $50 \mathrm{~cm}$ surrounded both sides with the rock model M1 (see Fig. 2)
Figure 8 shows the distributions of the spectral components forming the detector response representative for the anomaly without artifacts, as shown in Fig. 2.

A characteristic reduction in the count numbers in both the energy groups of neutrons within the M6 layer is observed, which results in the shape of the anomaly, as presented in Fig. 9.

Figures 10 and 11 show an analogous example but for the anomaly disturbed by the artifacts (corresponding to Fig. 4). We observe the opposite behavior of the spectral components. The excess of neutrons of the E1 component within the layer is the cause of creation of the artifact.

Appearance of artifacts was observed only in the case of anomalies registered by the thermal neutron detector. The confirmation of this thesis is the neutron energy distributions

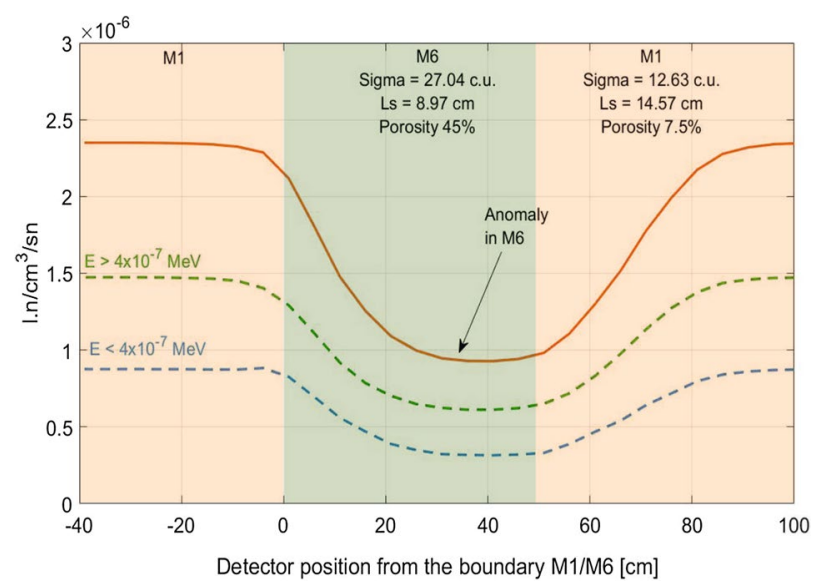

Fig. 9 Total response of the thermal neutron detector (continuous curve) corresponding to the anomaly in the M6 rock model surrounded by M1. The dotted lines show the contribution of neutrons with energies below and above $10^{-7} \mathrm{MeV}$

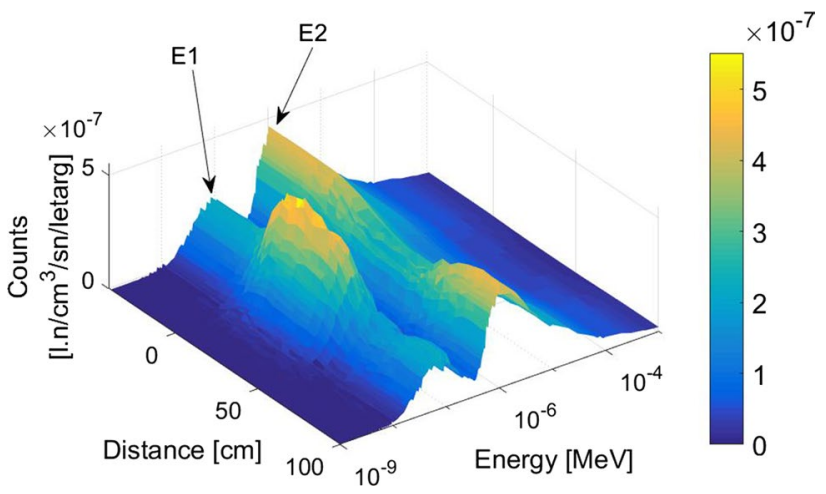

Fig. 10 Neutron energy-spatial distribution forming the response of the near thermal detector that registers the anomaly for the rock model M3 of thickness $50 \mathrm{~cm}$ surrounded both sides with the rock model M2 (see Fig. 4) 


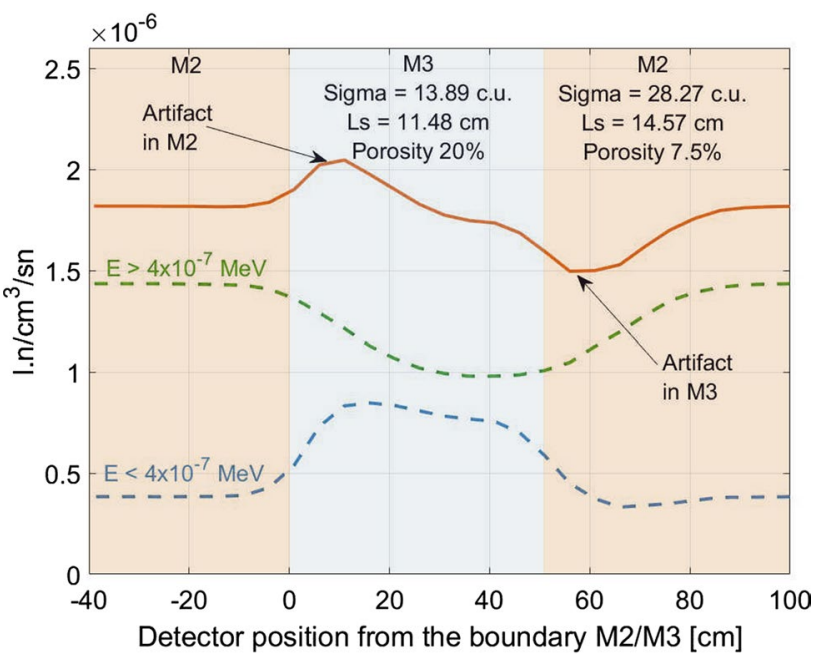

Fig. 11 Total response of the thermal neutron detector (continuous curve) corresponding to the anomaly in the M3 rock model surrounded by M2. The dotted lines show the contribution of neutrons with energies below and above $10^{-7} \mathrm{MeV}$

registered by epithermal neutron detectors. Figure 12 shows the neutron energy components of the response of these detectors for the example of anomaly bearing an artifact. (The example corresponds to Fig. 11: rock model M3 surrounded with M2.) Here, the neutron energy distributions have a consistent course, which results in the monotonic course of the anomaly.

\section{The presence of artifacts versus water content in the pores of rock samples}

The presence of artifacts near the boundary of layers, where neutron transport takes place, has been explained by the basic neutron properties of bordering rock media. The main focus was on two basic neutron transport parameters $L_{\mathrm{s}}$ and $\Sigma_{\mathrm{a}}$ of the geological formation analyzed. The purpose of interpretation of neutron probe signals is to obtain data on petrophysical parameters of the studied medium like porosity or reservoir water content. These petrophysical parameters are a complex combination of basic neutron parameters. This is evident in the neutron probe calibration procedure. For example, the semiempirical calibration method of neutron probes is a two-step procedure (Czubek 1994; Wiącek and Woźnicka 2016). In the first step, the probe's signal is correlated with the general neutron parameter GNP (it is a function of, among others, $L_{\mathrm{s}}$ and $\Sigma_{\mathrm{a}}$ ), and then, from the determined GNP value, the porosity of the medium for the given lithology and measurement conditions (diameter of borehole, etc.) is calculated. In our simplified examples, we assumed that pores are completely filled with water and porosity is here uniquely correlated with the water content.

Therefore, it is not possible to indicate an unequivocal relationship between the occurrence of artifacts that meet the conditions ( $3 \mathrm{a}$ or $3 \mathrm{~b}$ ) and the water content in the pores. This is due to the fact that water does not have to be a dominant factor on the absorption cross-sectional value of the rock medium. If we take into account the geological formation as a system of two main components: a rock matrix and reservoir water that completely fill the pores, then the $\Sigma_{\mathrm{a}}$ of such a medium is expressed as:

$\Sigma_{\mathrm{a}}=(1-\phi) \Sigma_{\mathrm{a} \text { matrix }}+\phi \Sigma_{\mathrm{w}}$

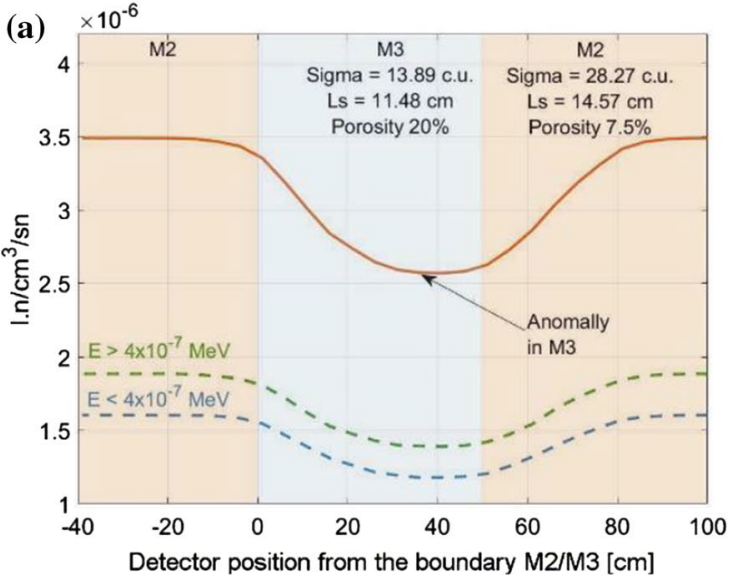

Fig. 12 Total response of the epithermal neutron detectors (continuous curve) corresponding to the anomaly in the M3 rock model surrounded by M2. The dotted lines show the contribution of neutrons

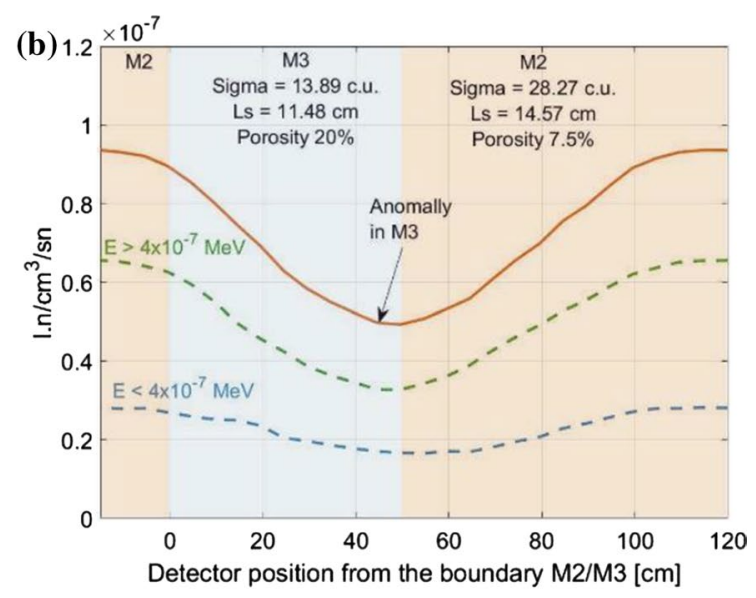

with energies below and above $10^{-7} \mathrm{MeV}$. a Near epithermal neutron detector and $\mathbf{b}$ far epithermal neutron detector 


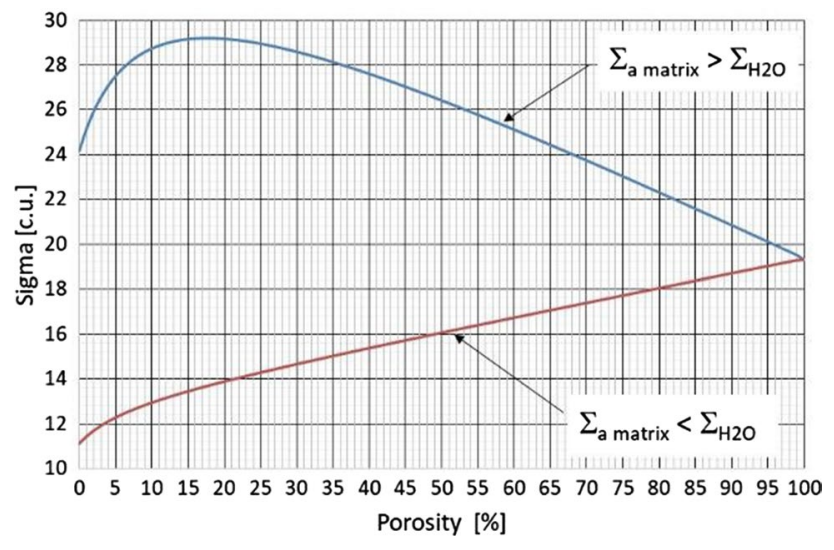

Fig. 13 Thermal neutron absorption cross section for rock matrix M (Table 2) of $\Sigma_{\text {a matrix }}=11.1$ and 24.2. c.u. in function of porosity. Pores are saturated with water: $\Sigma_{\mathrm{w}}=\Sigma_{\mathrm{H}_{2} \mathrm{O}}=19.30$ c.u.

where $\Sigma_{\mathrm{a} \text { matrix }}$ and $\Sigma_{\mathrm{w}}$ are thermal neutron absorption cross sections of the rock matrix and reservoir water, respectively, and $\phi$ is the porosity.

The shape of the function $\phi\left(\Sigma_{\mathrm{a}}\right)$ depends on the relation between $\Sigma_{\mathrm{a} \text { matrix }}$ and $\Sigma_{\mathrm{w}}$. The example of the $\phi\left(\Sigma_{\mathrm{a}}\right)$ function for the $M$ rock matrix (Table 2) of $\Sigma_{\text {a matrix }}=11.1$ c.u. and 24.2. c.u. is presented in Fig. 13.

Where $\Sigma_{\text {a matrix }}>\Sigma_{\mathrm{H}_{2} \mathrm{O}}$ is observed that in the range of low porosity, the influence of the rock matrix is dominant on the value of $\Sigma_{\mathrm{a}}$ which increases with porosity. Only for values of porosity higher than $17 \%$, the influence of water becomes dominant and $\Sigma_{\mathrm{a}}$ decreases to the value for water at $\phi=100 \%$. Such variability of $\phi\left(\Sigma_{\mathrm{a}}\right)$ indicates that for the same rock matrix we can have the same value of $\Sigma_{\mathrm{a}}$ at different porosities (e.g., here $\Sigma_{\mathrm{a}}=28$ c.u. for $\phi=6$ and 38\%).

For cases where artifacts were observed (2a-2d), the layer has higher porosity than the surrounding; thus, it contains more water in the pores. At the same time, these layers have much lower $\Sigma_{\mathrm{a}}$ than the surroundings. However, it cannot be assumed that the higher water content in the layer than in its surrounding causes the creation of an artifact near the border of the media. This is negated by the example given in (3a), where the difference in water content between the layer and the environment is significant (20\% and $45 \%$ ) and the artifact does not occur. In this case, despite the significant difference in water content, the difference between $\Sigma_{\mathrm{a}}$ is small. This confirms our conclusion that the unambiguous reason of forming artifacts is significant differences in the $L_{\mathrm{s}}$ and $\Sigma_{\mathrm{a}}$ values between bordered media.

\section{Conclusions}

The aim of the work was to show that the appearance of artifacts near the boundary of layers results from physical phenomena occurring during the transport of neutrons in that region. The appearance of artifacts is caused by a specific difference in values of parameters of bordering media for both fast neutrons $\left(L_{\mathrm{s}}\right)$ and thermal neutrons $\left(\Sigma_{\mathrm{a}}\right)$. The phenomenon of the appearance of artifacts is strongly related to the shape of the neutron energy-spatial distribution near the boundary of layers. The cases presented in this paper are for one selected type of the source and for one fixed source-detector distance. Both mentioned cases may have an impact on the shape and course of detector anomalies and the appearance of artifacts.

It was shown that artifacts appear only in the case of the anomalies registered by the thermal neutron detector for specific cases. Appearance of artifacts was observed only when both the neutron parameters: the thermal neutron absorption cross section $\left(\Sigma_{\mathrm{a}}\right)$ and the slowing down length $\left(L_{\mathrm{s}}\right)$, of one medium differ by at least $20 \%$ from those of the other medium.

The presence of artifacts on the neutron profiling curve may be a cause of interpretation errors. Particularly within thin layers, the artifact can be misinterpreted as the layer response, whereas the actual anomaly has in fact an opposite course.

Acknowledgements Paper was presented at the CAGG 2019 Conference "Challenges in Applied Geology and Geophysics" organized at the AGH University of Science and Technology, Krakow, Poland, 10-13 September 2019.

Open Access This article is distributed under the terms of the Creative Commons Attribution 4.0 International License (http://creativeco mmons.org/licenses/by/4.0/), which permits unrestricted use, distribution, and reproduction in any medium, provided you give appropriate credit to the original author(s) and the source, provide a link to the Creative Commons license, and indicate if changes were made.

\section{References}

Czubek JA (1994) Neutron tool calibration by a scaling procedure. Nucl Geophys 8:26

Gardner RP, Xu L, Wang J (2007) Some lessons learned from MCNP usage. In: SPWLA 48th annual logging symposium, June 3-6 (2007) pap. K

MCNP Team (2008) X-5 Monte Carlo Team, MCNP—a General Monte Carlo N-Particle Transport Code Version 5. Los Alamos National Laboratory LA-UR-03-1987

Wiaccek U (2018) Application of computer modeling and simulations to the development of the nuclear geophysical measurement methods using neutron well logging. IFJ PAN, pp 1-199, ISBN 978-83$63542-02-3$ (in polish)

Wiącek U, Dworak D (2018) Preliminary analysis of the artifacts appearing on the NNTE probe response curves at layers boundary of the two-layer rock media. In Polish, Mat. Międzynarodowej Konf. Naukowo-Technicznej Geopetrol 2018, Development of hydrocarbon exploration and production technologies. Instytut Nafty i Gazu, Państwowy Instytut Badawczy, pp 255-263, ISBN 978-83-65649-27-0

Wiącek U, Woźnicka U (2016) Feasibility of Monte Carlo modelling for the neutron-neutron logging tool response in specific 
geological models. Geol Geophys Environ 42:365-383. https:// doi.org/10.7494/geol.2016.42.3.365

Woźnicka U, Dworak D, Wiącek U, Zorski T (2012) Geofizyczne profilowania neutronowe w asymetrycznych formacjach cienkowarstwowych (Symulacje komputerowe na przykładzie sondy NNTE). In: IFJ PAN, Kraków, ISBN: 978-83-934248-7-0 (in polish) 